\title{
Acute fasting diminishes the circadian rhythm of biochemical markers of bone resorption
}

\author{
A Schlemmer and C Hassager \\ Center for Clinical and Basic Research, Ballerup Byvej 222, DK-2750 Ballerup, Denmark \\ (Correspondence should be addressed to A Schlemmer)
}

\begin{abstract}
Objective: Biochemical markers of bone turnover exhibit circadian rhythms with the peak during the night/early morning and the nadir in the late afternoon. The nocturnal increase in bone resorption could theoretically be caused by the absence of food consumption which brings about a decrease in net calcium absorption and an increase in parathyroid hormone (PTH), followed by increased bone resorption in response to the body's demand for calcium. The aim of the present study was to assess the influence of a 33-h fast on the circadian variation in biochemical markers of bone turnover.

Design: Eleven healthy premenopausal women (age: $24 \pm 5$ years) participated in a randomised, crossover study consisting of two periods: either $33 \mathrm{~h}$ of fasting (fasting) followed 1 week later by a 33-h period with regular meals eaten at $0800-0830 \mathrm{~h}, 1130-1230 \mathrm{~h}$ and $1800-1900 \mathrm{~h}$ (control) or vice versa.

Methods: Urinary CrossLaps (U-CL/Cr) corrected with creatinine, as a marker of bone resorption; serum osteocalcin (sOC) as a marker of bone formation; serum intact PTH (iPTH); serum phosphate; and serum calcium corrected with albumin.

Results: Both the fasting and the control periods showed a significant circadian rhythm in U-CL/Cr $(P<0.001)$, but the decrease was significantly less pronounced in the morning hours during the fasting period. Fasting resulted in a significant decrease in serum iPTH (throughout the study period) as compared with the control period $(P<0.05-0.001)$. No change was observed in sOC by fasting. Conclusion: Food consumption has a small influence on the circadian variation in bone resorption, independent of PTH. The fall in iPTH during fasting may be secondary to an increased bone resorption produced by fasting.
\end{abstract}

European Journal of Endocrinology 140 332-337

\section{Introduction}

A well-known circadian variation with the peak during the night/early morning and the nadir late in the afternoon has been described in biochemical parameters of bone resorption such as the urinary excretion of pyridinium cross-links (1-4), urinary cross-linked N-telopeptide of type I collagen (NTx) (5), urinary CrossLaps (6) and serum carboxy-terminal pyridinoline cross-linked telopeptide of type I collagen (7). A less pronounced circadian variation is also known in biochemical markers of bone formation, such as serum osteocalcin (sOC) $(4,8)$ and serum procollagen type I carboxy-terminal propeptide $(7,9)$.

Whereas the circadian variation in bone formation may be dependent on the circadian fluctuation in serum cortisol (10-12), the aetiology of the circadian variation in bone resorption remains unknown. Earlier studies have found the variation in bone resorption to be independent of age, menopausal status, posture (2), and the circadian fluctuations in serum cortisol
(12). However, the influence of calcium is ambiguous. Suppression of intact parathyroid hormone (iPTH) with a constant infusion of calcium did not influence the circadian pattern of the urinary Ntx (5), whereas the nocturnal peak in the urinary Ntx was suppressed by $1000 \mathrm{mg}$ calcium administered at $2300 \mathrm{~h}$ for 14 days (13). A recent study demonstrated, in rats, that when solid food and liquid intake were divided into portions, the peak in bone resorption seen after food ingestion was blunted and the 24-h bone resorption decreased. After 30 days on this schedule an increase in bone mass was observed (14).

Ebeling et al. (15) had previously shown that the total 24-h urinary excretion of pyridinium cross-links increased with 4 days of calcium deprivation (dietary calcium (230 mg/day) and treatment with a calciumbinding agent). Grinspoon et al. (16), however, failed to find any significant response in the 24-h urinary excretion of pyridinium cross-links with 4 days of fasting. Neither of these studies, however, was designed to examine the effect of fasting on the circadian rhythm 
in biochemical markers of bone resorption. Theoretically, therefore, the nocturnal peak in bone resorption may be caused by the absence of food consumption, followed by an increased bone resorption in response to the body's demand for calcium. The aim of the present study was to investigate this hypothesis.

\section{Participants and methods}

\section{Participants}

Eleven healthy premenopausal women (age: $24 \pm 5$ years; height: $168 \pm 6 \mathrm{~cm}$; weight: $63 \pm 6 \mathrm{~kg}$ (mean \pm 1 s.D.)) participated in a randomised, cross-over study consisting of two periods of either $33 \mathrm{~h}$ of fasting (water, coffee and tea permitted) followed 1 week later by a 33-h period with regular meals eaten at 0800$0830 \mathrm{~h}, 1130-1230 \mathrm{~h}$ and $1800-1900 \mathrm{~h}$ or vice versa. Normal daytime activities and sleeping pattern were permitted. None of the women had known endocrine or renal disease, or were taking any medication at the time of the study.

All the women fasted from $2300 \mathrm{~h}$ on the evening before the start of the two 24-h periods. The participants emptied their bladder at $0500 \mathrm{~h}$ in the morning, and urine was collected in 3-h aliquots starting at $0500 \mathrm{~h}$ until the following day at $0800 \mathrm{~h}$. Blood samples were taken at 3-h intervals starting at $0800 \mathrm{~h}$. The blood and urine samples were stored at $-20{ }^{\circ} \mathrm{C}$ until assayed.

The research protocol was approved by the Ethical Committee of Copenhagen County (KA95114g). All the participants gave their informed consent, in accordance with the Helsinki II Declaration.

\section{Methods}

Urinary CrossLaps were measured by an ELISA assay based on an antiserum (rabbit) reaction with a sequence derived from type I collagen (EKAHDGGR). The average within-run and total coefficients of variation of the assay were $5.3 \%$ and $6.6 \%$ respectively (17). The results were then corrected with creatinine $(\mathrm{Cr})$ and expressed as $\mathrm{mg} / \mathrm{mol}$ creatinine.

The sOC was measured by a two-site enzymelinked immunosorbent assay (N-MID hOsteocalcin; Osteometer BioTech A/S, Herlev, Denmark). The assay is based on two highly specific monoclonal antibodies against human osteocalcin, recognising the mid region (amino acids 20-43) and the N-terminal region (amino acids 1-19) respectively, and the assay thus detected intact osteocalcin $(1-49)$ as well as the N-terminal mid fragment $(1-43)$. The assay had a measuring range of 5.0-75.0 $\mu \mathrm{g} / \mathrm{l}$ and a detection limit of $2.0 \mu \mathrm{g} / \mathrm{l}$. The intra- and interassay coefficients of variation were less than $7 \%(18)$.

Serum iPTH was measured by a commercially available IRMA (Coat-A-Count Intact PTH IRMA; Diagnostic Products Corporation, Los Angeles, CA,
USA). The detection limit was $0.1 \mathrm{pmol} / \mathrm{l}$ and the intra-assay coefficient of variation was $6.0 \%$.

Serum calcium, serum phosphate, urinary creatinine, and serum albumin were measured by standard laboratory methods.

\section{Statistical analysis}

The results of the biochemical assays were analysed using the procedures of the SAS Institute (19). To obtain normality and homogeneity of variance, urinary CrossLaps/Cr and sOC were logarithmically transformed (log concentration) before analysis of the circadian rhythm. These serial measurements were analysed according to concepts described by Matthews et al. (20). The 24-h cyclical changes were calculated for each woman by multiple-regression analysis, with sine and cosine as independent variables (e.g. $\mathrm{y}=\beta 1 \sin (t)+\beta 2 \cos (t)+\alpha$, where $t=2 \pi$ time $(\mathrm{h}) / 24)$. The coefficients $(\beta 1$ and $\beta 2$ ) to sine and cosine were then used as summary measures of the circadian variation and compared with zero by multiple analysis of variance (19). Student's paired $t$-test was used for comparison within groups. For visualising the effect of fasting, a delta value of urinary CrossLaps/ $\mathrm{Cr}$ and $\mathrm{sOC}$ is given as mean of control value-fasting value for each individual at each time-point.

Serum calcium was corrected by albumin (e.g. total $\mathrm{Ca}+1.1(0.70$-serum albumin $)$.

\section{Results}

Figure 1 shows the 24-h fluctuation in the urinary excretion of CrossLaps/Cr and sOC. A highly significant circadian variation in urinary CrossLaps/Cr was observed during both the fasting and the control period $(P<0.001)$. A significantly less pronounced decrease during the morning and afternoon hours $(P<0.05-0.01)$ was observed in the fasting group as compared with the controls. sOC showed a circadian rhythm during the control period $(P<0.01)$ and the fasting period $(P=0.05)$. Fasting did not change the circadian pattern in SOC significantly.

Figure 2 shows the 24-h fluctuation in the urinary excretion of CrossLaps $/ 3 \mathrm{~h}$. The pattern of the curves was similar to the CrossLaps/Cr curves, with a significantly less pronounced decrease observed in the fasting period between 1100 and $1400 \mathrm{~h}(P<0.01)$. One value was dismissed at the first time-point because of collection error.

Figure 3 shows the 24-h fluctuation in serum iPTH, serum calcium (corrected for albumin) and serum phosphate. Fasting significantly decreased $(P<0.05-$ $0.001)$ iPTH and abolished the circadian rhythm in iPTH. Albumin-corrected serum calcium was significantly higher during the daytime in the fasting period than in the control period. In serum phosphate no significant differences between the fasting and the control period was seen at any time-point. 

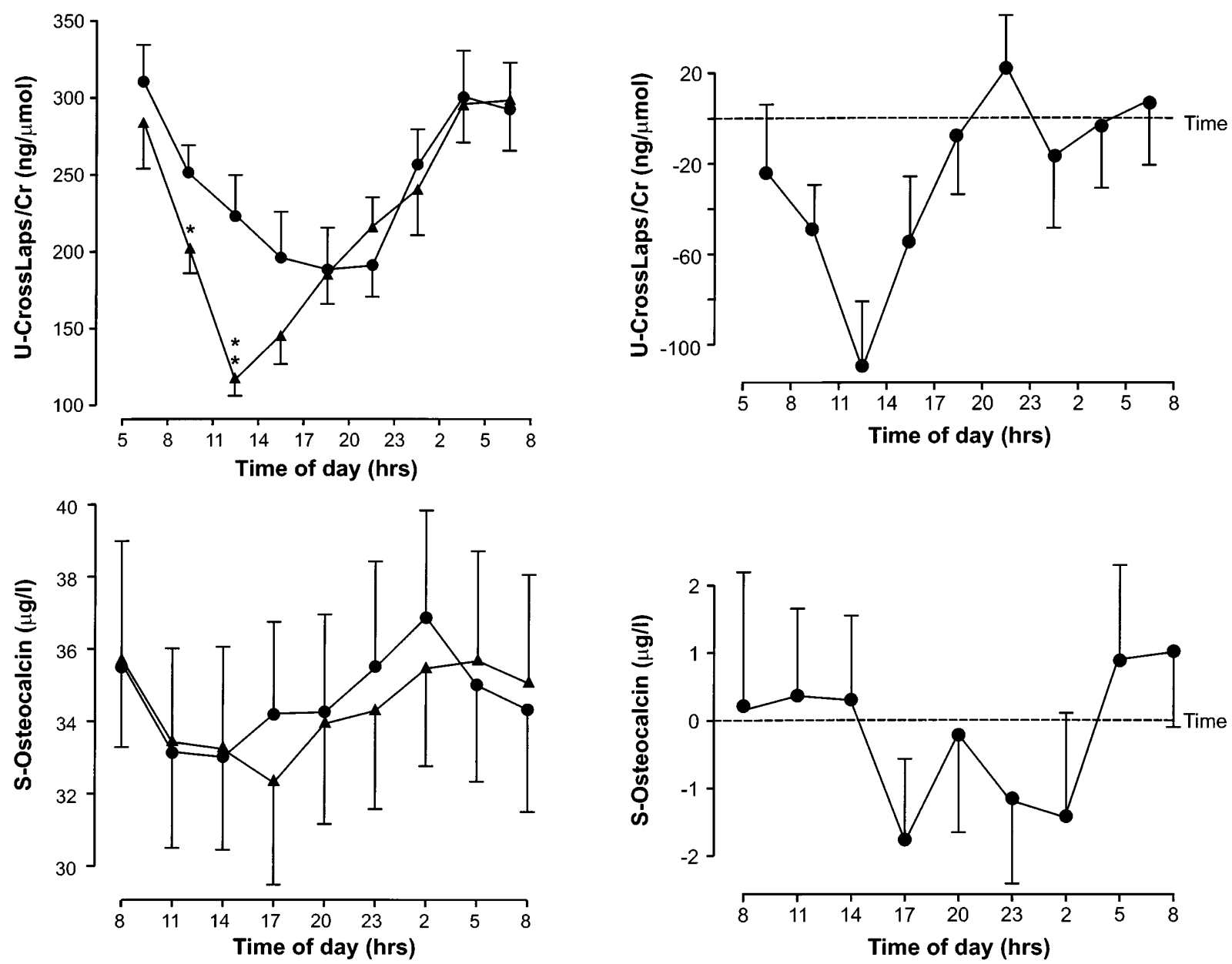

Figure 1 Mean values of the urinary $(U)$ excretion of CrossLaps/Cr and serum (S) osteocalcin during the two 24-h periods (left-hand figures). Fasting is shown as solid circles and control as solid triangles. Mean delta value of urinary $\mathrm{Cross} \mathrm{Laps} / \mathrm{Cr}$ and sOC is given as control value-fasting value for each individual at each time-point (right-hand figures). Values are means \pm S.E.M. ${ }^{*} P<0.05$; ${ }^{* *} P<0.01$.

The mean (average of eight time-points) increased significantly in serum calcium $(\mathrm{mmol} / \mathrm{l})$ (corrected for albumin $)(2.31 \pm 0.05$ vs $2.36 \pm 0.03 ; P<0.05)$ and decreased significantly in serum iPTH $(4.4 \pm 1.6$ vs $3.1 \pm 1.0 \mathrm{pmol} / \mathrm{l} ; \quad P<0.01)$ whereas no significant differences was observed in the urinary CrossLaps/Cr (220 \pm 59 vs $246 \pm 71 \mathrm{ng} / \mu \mathrm{mol}$; not significant) or serum phosphate $(1.36 \pm 0.12$ vs $1.34 \pm 0.09 \mathrm{mmol} / \mathrm{l}$; not significant). All values are given as control vs fasting.

\section{Discussion}

Food consumption alone cannot explain the circadian rhythm in the biochemical markers of bone resorption even though fasting significantly changes the circadian pattern. However, part of the circadian variation seen in healthy individuals is probably caused by food.

The circadian variation in the biochemical markers of bone resorption seen in this study was more pronounced in the control period, when regular

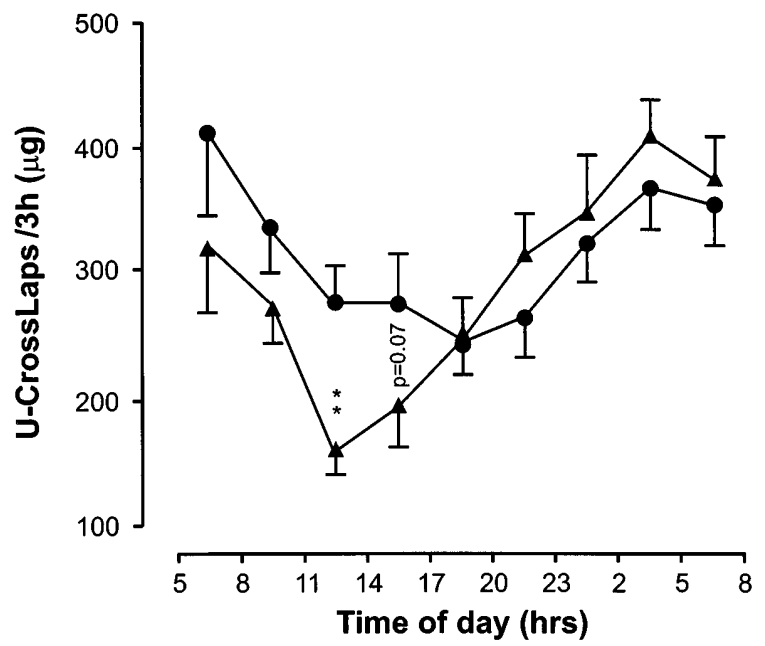

Figure 2 Mean value of the urinary $(\mathrm{U})$ excretion of Crosslaps $/ 3 \mathrm{~h}$ during the two 24-h periods. Fasting is shown as solid circles and control as solid triangles. Values are means \pm S.E.M. ${ }^{*} P<0.01$. 

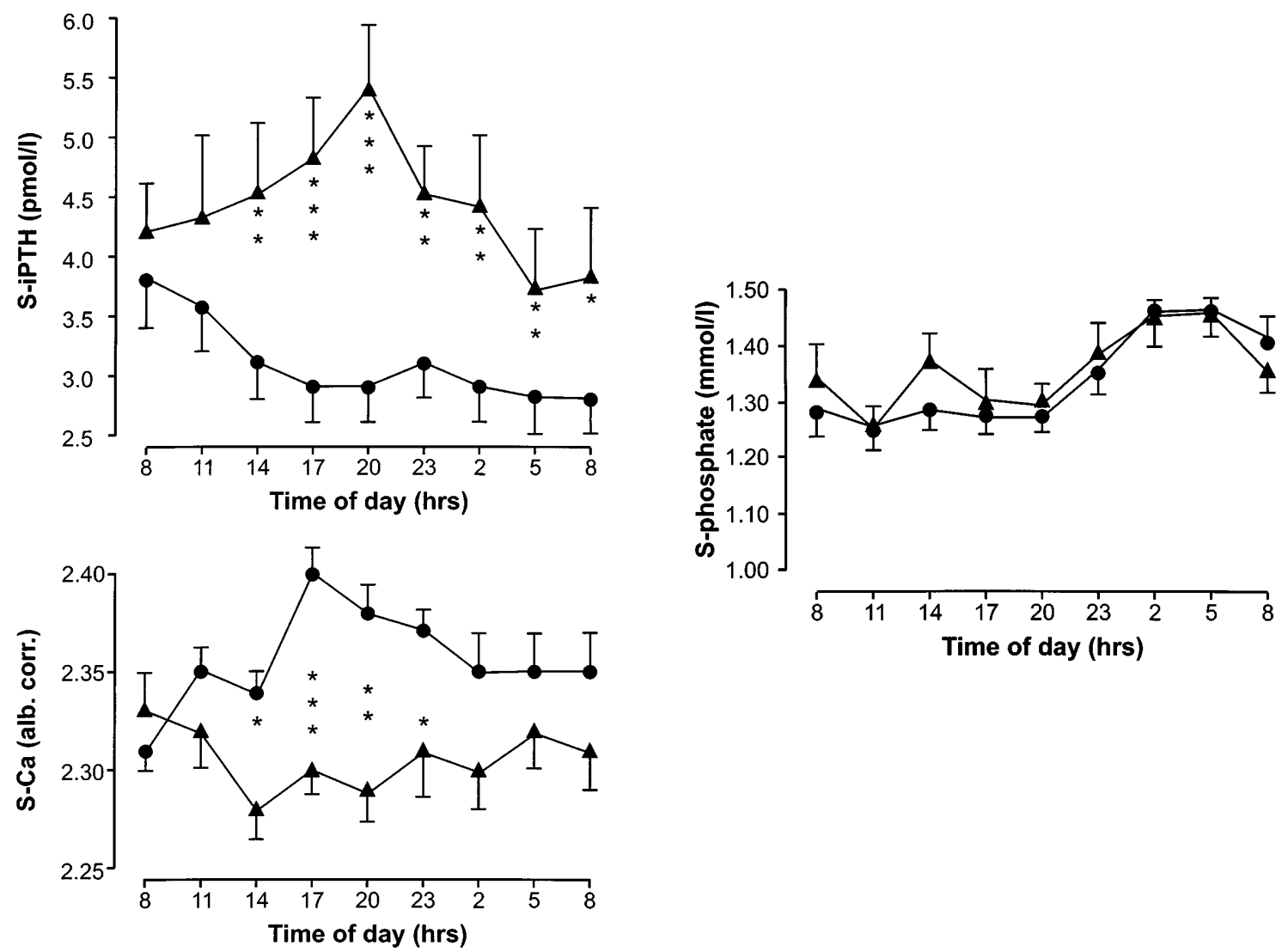

Figure 3 Mean value of serum (S) iPTH, serum calcium (S-Ca) (alb.corr., corrected for albumin) and serum (S) phosphate during the two 24 -h periods. Fasting is shown as solid circles and control as solid triangles. Values are means \pm S.E.M. ${ }^{\star} P<0.05 ;{ }^{* \star} P<0.01 ;{ }^{\star \star \star} P<0.001$.

meals were served, than in the fasting period. Urinary CrossLaps/Cr fell more significantly after food ingestion in the daytime, whereas the curves for the evening and the following night (when none of the participants were given any meals) were indistinguishable.

This effect on urinary CrossLaps/Cr was not caused by an increase in PTH in order to maintain the serum calcium at an acceptable physiological level, because serum iPTH actually fell and the circadian pattern was abolished, whereas serum calcium increased during fasting. It is more likely that a direct demineralisation took place during fasting, which increased serum calcium, followed by a decrease in serum iPTH as the outcome. These changes may be mediated by the acidosis caused by fasting. An earlier study by Kocian \& Brodan (21) demonstrated that short-term starvation of obese subjects induces metabolic acidosis and increased calciuria, resulting in a severe negative calcium balance. As most body calcium resides in bone mineral, the elevated urinary excretion of calcium is likely to originate from bone. Experimental studies with neonatal mouse calvariae cultured in medium with a reduced $\mathrm{pH}$ (an in vitro model of metabolic acidosis) have further shown that there is a net efflux of calcium from the bone during acute 3-h culture (22) and chronic culture $(24 \mathrm{~h}$ or longer) $(23,24)$. Furthermore, Krieger et al. (25) demonstrated increased osteoclastic activity and decreased osteoblastic activity in an experimental model of metabolic acidosis. Hence the well-known circadian variation in bone resorption with the peak during the night could, theoretically, be caused in part by the nightly fast - although the present study showed that this can only explain a small part of the circadian variation in bone resorption. In serum phosphate no significant difference was observed between the fasting and control period at any time-point, suggesting that the effect of fasting is mediated through calcium metabolism rather than phosphate.

When the present study was planned there were no data in the literature regarding the influence of food intake on the circadian variation of bone resorption. We thus did not know whether a change in food intake would affect the overall circadian variation in bone resorption or whether it would have an acute effect just during daytime or no effect at all. The present study was therefore designed to have $80 \%$ 
power (at a 5\% significance level) to detect an elimination of the circadian effect on urinary excretion of CrossLaps at a specific time-point. We found a significant effect of fasting only during the early day time which was not reflected in the 24-h urinary excretion of CrossLaps. We therefore believe that this finding needs to be confirmed before a final conclusion can be drawn regarding the magnitude of the effect of food intake on the circadian variation of bone resorption.

We did not include the participants at a specific time in their menstrual cycle in this study. However the time-span between the two study periods is short (1 week) and the study was performed as a randomised, cross-over study for which reason we do not believe that the fluctuations in hormones during the menstrual period has any influence on the results presented in this article.

Serum iPTH peaked at about $2000 \mathrm{~h}$ in our control period, whereas it usually occurs between 0200 and $0600 \mathrm{~h}$ (26-28). The participants were up in order to empty their bladder completely. This practice has no influence on the circadian variation in biochemical markers of bone resorption (2), but perhaps it affected the time at which serum PTH peaked. A shift in sleeping time is known to blunt the start of the rise in serum PTH (27), although the theoretical changes in sleeping pattern caused by the nightly awakening in this study are of less magnitude than in the study of Logue et al. (27).

We and others have shown the circadian rhythm in bone resorption to be independent of sex steroids, posture (2), and fluctuations in cortisol (12) and PTH (5). The present acute fasting study provides the first evidence to explain parts of the changes in the circadian variation in biochemical markers of bone resorption. However, it explains only a small part, the greater part of the aetiology of the circadian rhythm in bone resorption still remains unsolved. Further studies are needed - especially a longer period of fasting - in order to assess whether the observed changes continue in the following days, and to establish which particular components of the food are able to induce a further decrease in the urinary excretion of CrossLaps. The latter is probably not caused by calcium intake, based on the response of serum PTH to fasting.

In conclusion, we found that food consumption in the morning potentiates the morning/early afternoon decrease in the urinary excretion of CrossLaps, a new marker of bone resorption. This effect seems to be independent of PTH.

\section{Reference}

1 Schlemmer A, Hassager C, Jensen SB \& Christiansen C. Marked diurnal variation in urinary excretion of pyridinium crosslinks in premenopausal women. Journal of Clinical Endocrinology and Metabolism 199274 465-470.
2 Schlemmer A, Hassager C, Pedersen BJ \& Christiansen C. Posture, age, menopause, and osteopenia do not influence the circadian variation in the urinary excretion of pyridinium crosslinks. Journal of Bone and Mineral Research 19949 18831888.

3 Eastell R, Calvo MS, Burritt MF, Offord KP, Russell RGG \& Riggs BL. Abnormalities in circadian patterns of bone resorption and renal calcium conservation in type I osteoporosis. Journal of Clinical Endocrinology and Metabolism 1992 74 487-494.

4 Eastell R, Simmons PS, Colwell A, Assiri AMA, Burritt MF, Russell RGG et al. Nyctohemeral changes in bone turnover assessed by serum bone Gla-protein concentration and urinary deoxypyridinoline excretion: effects of growth and ageing. Clinical Science 199283 375-382.

5 Ledger GA, Burritt MF, Kao PC, O’Fallon WM, Riggs BL \& Khosla S. Role of parathyroid hormone in mediating nocturnal and age-related increases in bone resorption. Journal of Clinical Endocrinology and Metabolism 199580 3304-3310.

6 Schlemmer A, Ravn P, Hassager C \& Christiansen C. Morning or evening administration of nasal calcitonin? Effects on biochemical markers of bone turnover. Bone 199720 63-67.

7 Hassager C, Risteli J, Risteli L, Jensen SB \& Christiansen C. Diurnal variation in serum markers of type I collagen synthesis and degradation in healthy premenopausal women. Journal of Bone and Mineral Research 19927 1307-1311.

8 Nielsen HK, Brixen K \& Mosekilde L. Diurnal rhythm and 24-hour integrated concentrations of serum osteocalcin in normals: influence of age, sex, season, and smoking habits. Calcified Tissue International 199047 284-290.

9 Pedersen BJ, Schlemmer A, Rosenquist C, Hassager C \& Christiansen C. Circadian rhythm in type I collagen formation in postmenopausal women with and without osteopenia. Osteoporosis International 19955 472-477.

10 Nielsen HK, Brixen K, Kassem M, Charles P \& Mosekilde L. Inhibition of the morning cortisol peak abolishes the expected morning decrease in serum osteocalcin in normal males: evidence of a controlling effect of serum cortisol on the circadian rhythm in serum osteocalcin. Journal of Clinical Endocrinology and Metabolism 199274 1410-1414.

11 Nielsen HK, Charles P \& Mosekilde L. The effect of single oral dose of prednisone on the circadian rhythm of serum osteocalcin in normal subjects. Journal of Clinical Endocrinology and Metabolism 198867 1025-1030.

12 Schlemmer A, Hassager C, Alexandersen P, Fledelius C, Pedersen BJ, Kristensen JØ et al. The circadian variation in bone resorption is not related to serum cortisol. Bone 199721 83-88.

13 Blumsohn A, Herrington K, Hannon RA, Shao P, Eyre DR \& Eastell R. The effect of calcium supplementation on the circadian rhythm of bone resorption. Journal of Clinical Endocrinology and Metabolism 199479 730-735.

14 Mühlbauer RC \& Fleisch $\mathrm{H}$. The diurnal rhythm of bone resorption in the rat. Effect of feeding habits and pharmacological inhibitors. Journal of Clinical Investigation $1995951933-$ 1940.

15 Ebeling PR, Jones JD, Burritt MF, Duerson CR, Lane AW, Hassager $\mathrm{C}$ et al. Skeletal responsiveness to endogenous parathyroid hormone in postmenopausal osteoporosis. Journal of Clinical Endocrinology and Metabolism 199275 1033-1038.

16 Grinspoon SK, Baum HBA, Kim V, Coggins C \& Klibanski A. Decreased bone formation and increased mineral dissolution during acute fasting in young women. Journal of Clinical Endocrinology and Metabolism 199580 3628-3633.

17 Bonde M, Qvist P, Fledelius C, Riis BJ \& Christiansen C. Immunoassay for quantifying type I collagen degradation products in urine evaluated. Clinical Chemistry 199440 2022-2025.

18 Rosenquist C, Quist P, Bjarnason N \& Christiansen C. Measurement of a more stable region of osteocalcin in serum by ELISA with two monoclonal antibodies. Clinical Chemistry 199541 1439-1445 
19 SAS Institute. SAS/STAT Guide for Personal Computers, ed. 6. Cary, NC: SAS Institute, 1987.

20 Matthews JNS, Altman DG, Campbell MJ \& Royston P. Analysis of serial measurements in medical research. British Medical Journal $1990300230-235$.

21 Kocian J \& Brodan V. Simultaneous correction of Ca deficiency and acidosis in fasting obese patients as a prevention of bone demineralisation. Nutrition and Metabolism 197923 391-398.

22 Bushinsky DA, Krieger NS, Geisser DI, Grossman EB \& Coe FL. Effect of $\mathrm{pH}$ on bone calcium and proton fluxes in vitro. American Journal of Physiology (Renal Fluid Electrolyte Physiology 14) 1983 245 F204-F209.

23 Bushinsky DA. Net calcium efflux from live bone during chronic metabolic, but not respiratory, acidosis. American Journal of Physiology (Renal Fluid Electrolyte Physiology 25) 1989256 F836-F842.

24 Chabala JM, Levi-Setti R \& Bushinsky DA. Alteration in surface ion composition of cultured bone during metabolic, but not respiratory, acidosis. American Journal of Physiology (Renal Fluid Electrolyte Physiology 30) 1991261 F76-F84.

25 Krieger NS, Sessler NE \& Bushinsky DA. Acidosis inhibits osteoblastic and stimulates osteoclastic activity in vitro. American
Journal of Physiology (Renal Fluid Electrolyte Physiology 31) 1992 262 F442-F448.

26 Logue FC, Fraser WD, Gallacher SJ, Cameron DA, O’Reilly DSt], Beastall GH et al. The loss of circadian rhythm for intact parathyroid hormone and nephrogenous cyclic AMP in patients with primary hyperparathyroidism. Clinical Endocrinology 1990 32 475-483.

27 Logue FC, Fraser WD, O'Reilly DStJ, Christie J, Cameron DA, Wallace DC et al. Sleep shift dissociates the nocturnal peaks of parathyroid hormone (1-84), nephrogenous cyclic adenosine monophosphate, and prolactin in normal men. Journal of Clinical Endocrinology and Metabolism 199275 2529.

28 Fraser WD, Logue FC, Christie JP, Cameron DA, O'Reilly DStJ \& Beastall GH. Alteration of the circadian rhythm of intact parathyroid hormone following a 96-hour fast. Clinical Endocrinology $199440523-528$.

Received 22 May 1998

Accepted 14 January 1999 